\title{
Téoros
}

Revue de recherche en tourisme

\section{Le plan de développement touristique de Charlevoix : un pari environnemental}

\section{Pierre Tremblay}

Volume 10, numéro 1, mars 1991

Tourisme et environnement

URI : https://id.erudit.org/iderudit/1079813ar

DOI : https://doi.org/10.7202/1079813ar

Aller au sommaire du numéro

Éditeur(s)

Université du Québec à Montréal

ISSN

0712-8657 (imprimé)

1923-2705 (numérique)

Découvrir la revue

Citer cet article

Tremblay, P. (1991). Le plan de développement touristique de Charlevoix : un pari environnemental. Téoros, 10(1), 11-15. https://doi.org/10.7202/1079813ar d'utilisation que vous pouvez consulter en ligne.

https://apropos.erudit.org/fr/usagers/politique-dutilisation/ 


\section{Le plan de développement touristique de Charlevoix: un pari environnemental}
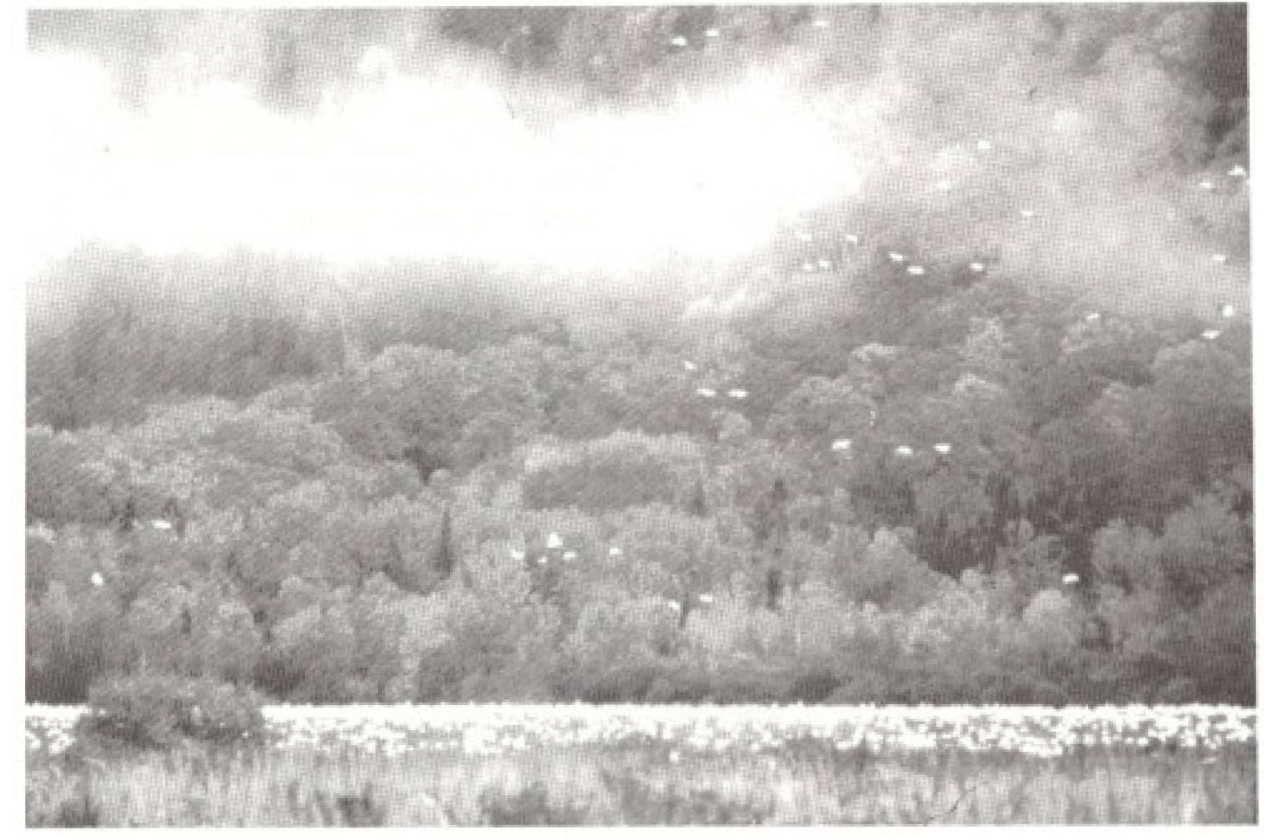

\section{Introduction}

En décembre 1987, l'Association touristique régionale de Charlevoix (ATRC) déposait son plan de développement touristique lors d'un colloque réunissant quelque cent cinquante intervenants de la région. Cetévénement visait à faire le bilan de deux années d'efforts de concertation, et permettait aux gens de Charlevoix de réaliser l'importance de l'élément naturel dans la constitution de leur produit touristique.

En effet, depuis 1985, l'ATRC, agissant à titre de maître d'oeuvre pour la réalisation du plan, avait réuni, au sein d'un comité de gestion, plusieurs organismes régionaux concernés par le développement touristique en plus de divers ministères sectoriels tels quel'OPDQ, le MLCP, le MTO et le MEIR. Au cours des étapes de confection du plan, plusieurs tournées de consultation publique ont été effectuées dans les cinq zones de Charlevoix afin de discuter des orientations mises de l'avant dans le plan de développement.

Comme le plan de développement touristique avait soulevé l'enthousiasme et fait l'objet d'un consensus de la part des per-

Monsieur Pierre Tremblay est directeur genéral de l'Asscciation touristique de Charlewoix. sonnes présentes au colloque, l'ATRC décidait d'entreprendre, dans les plus brefs délais, la mise en oeuvre de la stratégie sous-jacente au plan.

Nature et culture sont deux réalités indissociables de la région de Charlevoix. Elles prennent leurs racines dans les paysages de la côte escarpée ou de l'arrière-pays montagneux, dans unerichesse historique reliée à la villégiature multicentenaire ainsi que dans les diverses facettes de l'expression artistique.

Si on prend pour acquis qu'une région ne peut se développer et se promouvoir sur la base exclusive de son réseau d'hébergement, il devient dès lors impératif de développer et de structurer le produit touristique pour lequel la clientèle de séjour ou de passage pourrait manifester un intérêt. À partir de ce constat préliminaire, il nous faut convenir de l'importance de la définition d'une image cohérente à laquelle s'articule le développement des attraits régionaux. En outre, cette image devrait $s$ 'appuyer sur les caractéristiques spécifiques à la région qui démarquent Charlevoix des autres régions touristiques du Québec.

Vers 1985, la Commission canadienne de I'UNESCO avait formulé un projet decréa- tion d'une Réserve de la Biosphère dans la vaste zone verte ceinturant la ville de Québec. Par ce projet, elle visait à représenter l'écosystème de la forêt boréale mixte au sein du réseau international des zones naturelles protégées. Les démarches entreprises à ce moment dirigeaient le projet vers la Forêt Montmorency.

L'ampleur et la diversité des phénomènes écologiques qu'on rencontre dans Charlevoix font de cette région un des joyaux du patrimoine mondial, l'ATRC convint alors que ce statut était essentiel à la région. En effet, une fois obtenu, ce statut pourrait nous aider à réaliser une mise en valeur rationnelle des divers sites naturels que compte le territoire charlevoisien, en plus $\mathrm{d}^{\prime}$ assurer la mise en application du principe du développement durable à l'échelle régionale.

Lors d'une rencontre qui a eu lieu à Paris le 18 novembre 1988 , les responsables du programme "L'homme et la Biosphère" relevant de 1'UNESCO reconnaissaient Charlevoix à titre de Réserve de la Biosphère. En conséquence, cette région, où l'on retrouve un agencement majestueux de mer et de montagnes, fait dorénavant partie du prestigieux réseau mondial des Réserves de la Biosphère qui en compte 290 dans quelque 80 pays. Chacune de ces Réserves constitue un échantillon représentatif d'un des grands écosystèmes du monde; on y allie conservation et développement.

La nouvelle Réserve de la Biosphère de Charlevoix englobe plusieurs sites naturels: le pare des Grands-Jardins et ses caribous, le parc régional des Hautes-Gorges de la rivière Malbaie et ses paysages fabuleux ainsi que le Centre écologique de Port-au-Saumon et le Centreéducatif forestier les Palissades avec leurs écosystèmes protégés.

Après deux ans d'efforts soutenus, au cours desquels l'ATR de Charlevoix a investi plus de 50000 \$ en services professionnels tout en assumant un rôle prépondérant dans ce dossier majeur, l'UNESCO reconnaissait non seulement le caractère exceptionnel đu territoire concerné mais également "l'effort considérable pour assurer la parti- 
cipation des populations locales à $l^{7}$ utilisation rationnelle de l'espace et de ses ressources".

Ce dossier a done vu son aboutissement grâce aux efforts de concertation mis de l'avant par les divers partenaires socioéconomiques de Charlevoix. De plus, les succès obtenus en ce dossier confirment que le développement régional peut pren= dre sa source dans le dynamisme des intervenants régionaux unis dans une vision commune et appuyés, dans ce cas précis, par d'imminentes personnalités du monde scientifique, tel M. Pierre Dansereau, écologiste de réputation internationale.

Mentionnons enfin que l'ATRC poursuit son action dans ce dossier de développement en étant fortement représentée, ainsi que ses proches collaborateurs, au sein de la Corporation de la Réserve de la Biosphère de Charlevoix. Le defi futur sera de faireen sorte de stimuler les activités reliées à la bonne marche de la Réserve en travaillant. au mieux-être des collectivités locales tout en ayant à coeur la qualité de vie des citoyens de Charlevoix.

\section{Hautes-Gorges de la rivière Malbaie}

Afin de rékaliser le défi d"une Réserve de la Biosphère fonctionnelle et active, il fallait que ses aires centrales soient bien développees: protection et mise en valeur formant un tout indissociable.

Or, le vaste territoire des Hautes-Gorges de la rivière Malbaie $\left(233 \mathrm{~km}^{2}\right)$ n'était qu'un territoire non organisé (TNO) en 1986, après l'abandon du projet de parc par le gouvernement du Québec. L'ATRC prit donc l'initiative d'aller explorer le territoire à quelques reprises avec certains membres de son conseil d'administration. On décida alors d'y aménager un produit de type "parc" afin de suppléer l'absence du ministere du Loisir, de la Chasse et de la Pêche. A l'automne 1987, I'ATRC formait $1^{\prime}$ Association de développement des Hautes-Gorges de la rivière Malbaie et invitait les đeux municipalités régionales de comté (MRC) de Charlevoix-Est et Charlevoix ày siéger.

Après l'assemblée de fondation, on intéressa des investissseurs à la possibilité d'acheter un bateau mouche qui offrirait des croisieres sur la Malbaie, dans le secteur des Hautes-Gorges. L'ÁTRC trouva un bateau à Toronto ainsi qu'un groupe d'hommes d"affaires prêts à investir 150 $000 \$$ pour implanter ce serviced'excursion original.
Lors d'un colloque sur le développement

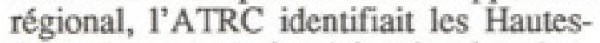
Gorges comme projet régional majeur dans son plan de développement touristique. Ce document estimait que le touriste n'est pas suffisamment mis en contactavec l'arrièrepays montagneux de Charlevoix. Pour pallier à cette carence, il recommandait donc que la zone de la Malbaie associât dorénavant son produit touristique aux ressources naturelles du site des HautesGorges. Deplus, ce développement permettrait à des localités comme Saint-Aimédes-Lacs de profiter davantage de l'achalandage touristique.

Au printemps 1988, la corporation des Hautes-Gorges négociait avec la Zone d'exploitation contrôlée (ZEC) des Martres un libre acces au territoire des HautesGorges. Ces négociations étant couronnées de succès, l'ATRC fournit gracieusement à la corporation une bâtisse pour servir de bâttiment d'accueil. Cette mềme année, la corporation obtenait diverses subventions totalisant près de $175000 \$ \mathrm{du}$ gouvernement fédéral pour procéder à l'aménagement de ce vaste territoire. $\mathrm{A}$ l'été, plus de 10000 personnes (3500 personnesen 1987) visitaient lesite. La corporation demanda en outre aux deux MRC de la région d'octroyer le statut de parc régionalau site des Hautes-Gorges, cequicorrespondait à l'affectation "conservation" déjà mise de l'avant dans leurs schémas d'aménagement respectifs.

Vers la fin del'année, la reconnaissance par l'UNESCO de Charlevoix en tant que Réserve de la Biosphère élevait le site des Hautes-Gorges au titre d'aire centrale de protection. Ce nouveau statut consacre la vocation de conservation de ce territoire qu'on peut considérer, à juste titre, comme l'un des plus beaux monuments naturels du Québec.

En 1989, le gouvernement du Québec octroyait une subvention de $24000 \$$ à la corporation qui bénéficiaitparailleursd'une autre tranche de subvention de 175000 \$ émanant du gouvernement fédéral. La fréquentation passait à près de 19000 personnes, dont la moitié environ avait utilisé les services du bateau d'excursion. Cette année-là, on assista à la mise en place d'un service de location de canots et de vélos de montagne et d'un service de restauration légère; de plus, on y aménagea un camping rustique et plusieurs sentiers de randonné.

Le parc régional des Hautes-Gorges a fait l'objet de nombreux reportages dans des revues et journaux prestigieux au pays et sur la scène internationale, tel le Figaro Magazine. Le site des Hautes-Gorges est devenu une image touristique de marque véhiculée un peu partout au Québec. En 1990 , la fréquentation connaissait une augmentation de $25 \%$, passant à pres de 25 000 personnes.

Divers travaux de mise en valeur ont êté réalisés comme l'agrandissement du chalet d'accueil, la réfection du camping et le développement du réseau de sentiers de randonnee. La corporation des HautesGorges lança également un dépliant promotionnel et deux brochures d'interprétation.

Le plan de développement touristique avait identifié un projet de parc relatif au territoire des Hautes-Gorges. Un statut de parc aurait plusieurs avantages, parmi lesquels la protection a long terme du site ainsi qu'une mise en valeur adéquate.

Or, s'il est apparu évident aux divers intervenantsde Charlevoix que le gouvernement du Québec n'avait pas l'intention d'y créer un parc provincial au cours des prochaines années, il en va autrement pour ce qui est d'un éventuel statut de parc fédéral. En effet, le gouvernement du Canada désire compléter son réseau de parcs nationaux et le site des Hautes-Gorges fait partie d'un vaste territoire où il souhaite créer un parc à brève échéance. Si on prend pour acquis

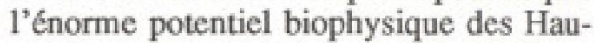
tes-Gorges et sa présence au sein d'une région touristique â caractère international comme Charlevoix, on peut considérer que les arguments ne manquent pas en faveur de la création d'un pare national.

\section{Parc des Grands-Jardins}

Situé au nord du village de Saint-Urbain, le territoire des Grands-Jardins, dont la superficie est de $310 \mathrm{~km}^{2}$, constitue un parc de conservation depuis 1981 . Ce secteur de Charlevoix estconsidéréc comme exceptionnel, de par la présence d'une formation végétale nordique (la pessière à cladonie) et d'une espece faunique subartique (le caribou). En effet, ce parc fait découvrir un paysage de taïga à seulement $120 \mathrm{~km}$ de Québec.

Pour l'amateur d'expéditions en nature, Charlevoix dispose d'un produit unique dans l'arrière-pays; le secteur des GrandsJardins correspond bien aux attentes et aux intérêts spécifiques de cette clientèle.

Comme nous venons de le voir, le parc des Grands-Jardins regorge de potentiels. Cependant, malgré de louables efforts soutenus par le passé avec des moyens modestes, on se devait d'améliorer les aménagements et les activités du parc tant sur le plan qualitatif que quantitatif. En outre, il apparaissait important que naissent 
une coopération et un esprit de partenariat entre le gouvemement du Québec et le milieu régional.

En 1989, à l'initiativede l'ATRC, des intervenants de Charlevoix se sont réunis au sein d'un nouvel organisme, la Société charlevoisienne de mise en valeur du parc des Grands-Jardins. Cet organisme réunit $1^{\prime}$ Association touristique de Charlevoix, la M.R.C. de Charlevoix, la Chambre de commerce de Charlevoix-Ouest et la municipalité de Saint-Urbain. Il vise à faire en sorte que le parc des Grands-Jardins puisse prendre un essor véritable en termes d'achalandage etqu'ilcontribue audéveloppementde l'industrie touristique régionale.

Après la mise sur pied de la Société des Grands-Jardins, il était devenu essentiel de donner des racines régionales à ce parc provincial. C'est ainsi que la corporation a rencontré les autorités du MLCP pour négocier un contrat de concession permettant la prise en charge de certaines infrastructures (chalets, camping, etc.) et de certaines activités (accueil, interprétation, etc.).

La volonté de collaboration grandissante des intervenants régionaux est aussi largement inspirée par de nouveaux outils qui permettront de mieux planifier $1^{\dagger}$ aménagement du territoire. Ainsi, le milieu dispose maintenant d'un schêma d'aménagement qui confirme la vocation de conservation du parc des Grands-Jardins etqui en laisse présager une utilisation plus polyvalente, notamment pour des fins de recreation.

C'est cependant avec le plan de développement touristique de Charlevoix que nous avons pu voir toute l'importance du parc des Grands-Jardins comme élément dynamique essentiel à la mise en valeur et au développement de la région. En effet, on peut y lire: "Au titre de l'intégration des zones au produit touristique régional, il appert que l'établissement de liens plus étroits entre la zone de Baie-Saint-Paul et son arrière-pays constituera l'un des principaux défis à relever par le plan de développement touristique de Charlevoix. Même si le secteur des Grands-Jardins devra conserver la personnalité qui lui est propre, il sera nécessaire de l'intégrer ă la zone littorale".

Le bilan qu'on peut tracer de la première saison d'opération de la Société charlevoisienne de mise en valeur du parc desGrandsJardins s'avère très positif. En effet, l'achalandage a augmenté de façon notable et on peut mentionner que le profil de la clientèle correspond, pour une large part, au segment hors-Québec du tourisme qui visite la région de Charlevoix. De plus, nous avons assisté à une diversification des activites pratiquées (randonnée pédestre. canot, vếlo).

\section{Parc marin Saguenay/ Saint-Laurent}

Les autorités gouvernementales canadiennes travaillent sur un projet de parc marin à la confluence du Saguenay et du SaintLaurent depuis près d'une quinzaine d'années. L'ATRC avait identifié la création de ce parc marin comme projet majeur dans son plan de développement touristique en 1987; il devenait alors important d'ajouter le poids régional afin d'accélérer les négociations entre les deux paliers du gouvernement.

Estimantce projet indispensable à la consolidation du pôle de Saint-Siméon, l'ATRC entreprit donc de faire naître un esprit de concertation entre les trois régions de la Haute-Côte-Nord, du Bas-Saguenay et de Charlevoix et créa la coalition pour le parc marin Saguenay/Saint-Laurent. Dès le départ, cette nouvelle corporation a pu comptersur desalliés précieux tels l'Union québécoise pour la conservation de la nature (UQCN), Greenpeace et le Fonds mondial pour la nature/Canada.

Le président du Fonds mondial pour la nature/Canada, M. Monte Hummel, est venu "saluer cette initiative régionale en faveur de la protection de certaines especes marines, dont le béluga. Il a souligné l'importance de la participation de la population locale en faveur d'un tel projet de parc marin. En effet, dès le départ, la coalition a pu compter dans ses rangs un nombre impressionnantde municipalitésetd'organismes voués à l'aménagement, au développement ou à la conservation des trois régions mentionnées plus haut.

La coalition a demandé aux agents fédéral et provincial de venir discuter du projet de parc marin sur la place publique afin que les citoyens soient mieux informés des véritables enjeux reliés à la mise en place de cette infrastructure majeure.

Parmi lés objectifs visés par l'ATR de Charlevoix dans ce dossier, on peut citer notamment, l'extention des limites du parc jusqu'à Cap-ă-1'Aigle, l'adoption du béluga du Saint-Laurent comme emblème animalier du parc marin, la mise en place à Baie-Sainte-Catherine d'infrastructures adéquates pour le départ des croisières d'observation des mammiferes marins ainsi que le développement et l'organisation de services touristiques et $d$ 'infrastructures récréatives dans la zone de Saint-Siméon.
Cette localité devrait d'ailleurs jouer le ròle d'un pôle majeur, considérant sa position stratégique par rapport au flot touristique qui se rendra dans le parc marin au cours des prochaines années.

Donc, après quelques années, l'ATR de Charlevoix a vu ses efforts de concertation au sein de la Coalition pour le parc marin couronnés de succès. En effet, le 6 avril 1990 , nous assistions à une cérémonie officialisant la création du parc marin du Saguenay en présence des ministres de 1 'Environnement du Canada et du Loisir, de la Chasse et de la Pêche du Québec.

\section{Quai de Baie-Sainte-Catherine}

Dans le territoire du parc marin Saguenay/ Saint-Laurent, une infrastructure sert de point de départ pour les croisières d'observation des baleines et sur le fjord du Saguenay: il s'agit du quai de Baie-SainteCatherine.

Le plan de développement touristique de Charlevoix avait mis en lumière le très fort potentiel des croisières aux baleines par rapport à la clientèle touristique qui fréquente la région. En effet, cette industrie a commencé ses activités il y a une dizaine $\mathrm{d}^{*}$ annces et a maintenant atteint une ${ }^{4 t}$ vitesse de croisière" fort intéressante pour culminer en 1990 avec près de 80000 personnes, dont plus de 45000 ont pris leur départ du quai de Baie-Sainte-Catherine.

Ainsi, ce village côtier de Charlevoix confirme et accentue son importance relative dans ce secteur d'activité, car il représente un point d'embarquement crucial, à cause de sa situation géographique sur la rive sud du Saguenay.

Le quai de Baie-Sainte-Catherine a vu sa vocation changer au cours de la demière décennie. En effet, depuis 1982 il n'existe plus aucune activité commerciale reliée au transport de marchandises à cet endroit. et,

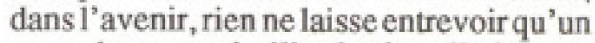
nouvel usager n'utilise les installations du quai de Baie-Sainte-Catherine pour la manutention de marchandises. Par contre, un consensus régional s'est manifesté au cours des dernières années pour que des améliorations importantes soient apportées aux installations portuaires de Baie-SainteCatherine pour les utiliser à des fins strictement touristiques.

On évalue les retombées économiques découlant de l'industrie des croisières d'observation des baleines et sur le fjord du Saguenay à près de quinze millions de dollars pour la région de Charlevoix. Par contre, la réalisation du projet de réfection 
des installations portuaires (quai, quais flottants, bâtiment d'accueil) pourrait entrẫner des retombées supplémentaires de plus de dix millions de dollars, ce qui ne manquera pas de favoriser l'essor économique de la région.

Les croisières d'observation des baleines ont permis de favoriser l'étalement saisonnier du tourisme, touten augmentant sensiblement les taux d'occupation des établissements hôteliers en basse saison (juin, septembre, octobre). Si l'industrie des croisières a permis de consolider le produit touristique de Charlevoix, elle contribue également à positionner la région comme destination internationale.

Au cours des prochaines années, on peut s'attendre à voir s'intensifier le développement des activités reliẹes à l'environnement et à la mise en valeur du Saint-Laurent à des fins touristiques: le projet de parc marin Saguenay/Saint-Laurent en est un bon exemple. La reconquête du fleuve, à peine amorcée, passe sans doute par des interventions importantes des divers paliers de gouvernement à titre de pourvoyeurs financiers.

Conscients des possibilités économiques énormes qu'offrent la consolidation et le développement de l'industrie des croisières sur le Saint-Laurent et le Saguenay, les divers intervenants de Charlevoix soutiennent que le gouvernement fédéral doit apporter sa contribution pour l'aménagement d'installations portuaires adéquates à Baie-Sainte-Catherine.

\section{Le Massif de Petite-Rivière-Saint- François}

Un autre dossier de toute première importance avait êté identifié dans le plan de développement touristique de Charlevoix en tant que projet à réaliser, c'est celui du Massif de Petite-Rivière-Saint-François. Encore là, $1^{\prime}$ A.T.R.C. a joué un rôle majeur comme organisme voué notamment au développement régional. L'ATRC est impliquée dans ce dossier depuis 1978; dès cette époque, elle a participé activement à la formation de la Société de développement du Massif avec le concours des municipalités.

La corporation, qui a formulé le concept de ski avec remontées en autobus, a vu le flot des études déferler les pentes (plus d'un demi-million de dollars) sans que cela n'apporte de résultats concrets. A l'origine, le projet se chiffrait à près de 300 millions de dollars. Au fil des ans, il s'est transformé en un projet plus modeste (mais sans doute plus réaliste) d'une quinzaine de millions de dollars.
Au cours de l'été 1990 , la société du Massif a amorcé des négociations avec le MLCP afin de conclure une ententeà l'hiver 1991. Mais, quoiqu'il advienne, il demeure sans conteste que le centre de ski du Massif est une station hors du commun, connue à travers l'Amérique du Nord et possédant la plus haute dénivelée de l'Est du Canada, soit plus de 800 mètres.

Le potentiel exceptionnel de ce domaine skiable unique au Québec a été mis en lumière par de nombreux articles percutants parus dans des médias prestigieux, tels Ski U.S., Ski Magazine et Powder. Touss'accordent sur la stratégie à mettre de l'avant: établir un corridor de ski regroupant le Mont-Sainte-Anne, le Massif de Petite-Rivière-Saint-François et le MontGrand-Fonds.

Dans le nouveau concept mis de l'avant par la société du Massif, on prévoit relier le secteur de La Malbaie au secteur de PetiteRivière au moyen d'une liaison ferroviaire "formule Bud". De plus, il nes'agirait plus d'une station quatre-saisons, mais d'une station conventionnelle (avec quelques remontées mécaniques) développée principalement à partir de la base de la montagne.

\section{Le secteur culturel}

Dans le secteur culturel, le plan de développement avait identifié quatre projets majeurs: la relocalisation du Musée régional à Pointe-au-Pic, l'implantation d'un Centre d'expositionà Baie-Saint-Paul, la construction d'un Pavillon de la Biosphère dans la région de Baie-Saint-Paul ainsi que la création d'une maison de la culture à SaintIrénée.

Nous pouvons d'ores et déjà souligner que le contexte créé par le plan de développement et l'ensemble des actions dynamiques entreprises par l'ATRC permettront de constituer un cadre de référence pour les quatre projets mentionnés plus haut.

\section{Le Musée de Charlevoix}

Le Musée de Charlevoix a été relocalisé dans de nouveaux locaux, bien situés par rapport au flux touristique, à Pointe-au-Pic. Cenouveau bâtimentanécessité un investissement de plus de deux millions et demide dollars, dont une bonne part provient des gouvernements fédéral et provincial. II comprend notamment plusieurs salles d'exposition, une zone d'accueil et une boutique, tandis que la mezzanine permet aux visiteurs d'avoir une vue exceptionnelle sur la rive de Charlevoix et le SaintLaurent.

\section{Le Centre d'exposition de Baie- Saint-Paul}

Le Centre d'exposition de Baie-Saint-Paul devrait se réaliser au cours des prochains mois. Ce centre deviendra alors un lieu de diffusion et de recherche unique au pays. Un nouvel immeuble moderne abritera un centre d'accueil, une boutique et diverses aires d'exposition. L'édifice actuel du Centre d'art seracomplètement réaménagé afin de permettrel'établissementd'un centre des métiers d'art. On y concentrera les activités liéesà la diffusion età la formation dans le domaine des métiers d'art.

\section{Le Pavillon de la Biosphère}

Pays de montagnes et de vallées où les formations géologiques exceptionnelles abondent, Charlevoix est connu pour la beauté de ses paysages. Pour illustrer ce potentiel unique et mieux faire connaître les implications relatives au statut de Réserve de la Biosphère, l'ATR de Charlevoix a élaboré un projet de Pavillon de la Biosphère. Estimé à près de trois millions de dollars, ce projet veut développer une image complémentaire forte mettant en lumière la diversité, la concentration et l'intérêt reconnu des phénomènes naturels observables dans Charlevoix. Une fois réalisé, il permettra d'engendrer une notoriété et une visibilité plus grandes sur la scène nationale et internationale.

Quatre thèmes principaux en composent l'essentiel de la thématique:

1- "Peindre le futur". Thème d'introduction visant à imaginer les répercussions de nos gestes sur le futur de notre environnement.

2- "Le Patrimoine mondial et les Réserves de la Biosphère". Constitue le corps principal de la thématique précisant des données multiples sur la Réserve de Charlevoix.

3- "Frayeurs de 1925". Simulation du tremblement de terre de 1925. Constitue l'attraction irrésistible sur laquelle est principalement fondée l'achalandage touristique. Informe les visiteurs tout en les rassurant sur les conséquences d'un tel séisme.

4. "La Place de 1'homme dans la Biosphère". Thème final mettant en relief l'importance de la population actuelle et des scientifiques qui vont encadrer la planification de la Réserve.

L'ATRC prévoit la réalisation du Pavillon de la Biosphère au cours de 1991. 


\section{La Maison de la Culture de Saint-Irénée}

Le Domaine Forget de Saint-Irénée jouit d'une réputation sans cesse grandissante dans les arts d'interprétation musicale. En plus d'assurer l'enseignement en musique et en danse, il présente de nombreux concerts au cours de la saison estivale. Les bấtiments actuels ne répondent plus aux besoins et un projet de l'ordre de 3,5 millions \$a été conçu.

Plus spécifiquement, celui-ci prévoit $1^{*}$ aménagement d'un amphithéâtre de près de 1000 places. Le nouvel édifice pourrait être construit sur un plateau situé à l'ouest du Domaine Forget et offrant une vue spectaculaire sur le Saint-Laurent.

Le conceptactuellement lélaboré laisse voir plusieurs particularités intéressantes. En effet, il a prếvu l'aménagement de 1000 places supplémentaires sur un parterre gazonné. L'ensemble de ces nouveaux équipements pourrait aider à structurer ce produit spécifique, tout en permettant la consolidation d'une infrastructure culturelle majeure.

\section{Autres actions}

Plusieurs autres réalisations ont découlé directement ou indirectement du plan de développement touristique de Charlevoix. On peut mentionner le dossier du traversier de l'île aux Coudres. Affectes gravement par l'instauration d'une tarification, les intervenants touristiques de l'île aux Coudres ont promptement réagi en demandant àl'ATRC d'intervenir auprès des instances politiques. Avec l'aide du ministre MarcYvan Côté, on a rétabli la gratuité sur le traversier, ce qui a permis d'accroitre la fréquentation de 35000 personnes pour atteindre 165000 visiteurs en 1990.

L'île aux Coudres constitue un attrait touristique en soi et on peut la considerer comme une destination touristique. $\AA$ A ce sujet, le plan de développement l'avait identifiée comme pôle d'appui de séjour. Cette réalité est importante, car elle conditionne et oriente la consolidation et la mise en place de differentes infrastructures touristiques.

Parmi les actions structurantes qui pourraient découler de l'analyse inhérente au plan de développement, on note l'aménagement d'une bande cyclable. Cet équipement était devenu une nécessitế, si on prend pour acquis la présence du nombre impresionnant de bicyclettes en location sur l'île. En ceinturant l'île aux Coudres, la bande cyclable rendrait accessibles les divers sites du circuit patrimonial.

\section{Charlevoix: une région performante}

Si la région touristique de Charlevoix est l'une des plus petites et des moins populeuses du Québec, ses performances sont élevées par rapport à la concurrence et témoignent de l'importance et du dynamisme du tourisme régional.

Charlevoix est la région du Québec qui retient le plus longtemps ses visiteurs (nuitées/voyage-personine) et occupe le premier rang pour les dépenses par voyage. Comparée à l'ensemble des autres régions touristiquesdu Québec, Charlevoix est l'une des régions qui compte le plus fort pourcentage de voyages d'agrément par rapport au total des voyages.

Globalement, la performance de Charlevoix au niveau du séjour et des dépenses est, pour une large part, attribuable au dynamisme de la commercialisation du produit touristique régional. À ce chapitre, mentionnons qu'en développant quelques produits régionaux, comme les auberges et les baleines, dont on estime qu'ils sont d'intérêt international, Charlevoix se distingue des autres régions touristiques du Québec et se prépare pour la conquête de nouveaux marchés.

Notons que l'offre d'hébergement dans Charlevoix est présente dans tous les villages, sous forme de gîtes du passant ou de chalets locatifs, et s'appuie sur un réseau d'auberges uniqueau payset plusieurs hôtels de villégiature, côtoyés par des motels offrant le confort traditionnel. Àcela, s'ajoute un réseau de terrains de camping de plus de 1000 sites et plusieurs pourvoiries permettent le sçjour en forêt, afin d'y pratiquer la chasse et la pêche.

En résumé, tous ces éléments de la trame touristique font de Charlevoix une région où l'on retrouve une forte concentration de produits touristiques de qualité, ce qui confirme l'envergure internationale decette destination.

\section{Conclusion}

Nous avons pu constater plus tôtque le plan de développement touristique de Charlevoix, déposé il y a plus de trois ans, a permis la réalisation de plusieurs projets moteurs. Dans certains cas, des corporations spécifiques ont insuffle leur dynamisme afin de concrétiser ces projets. Dans d'autres cas, c'est l'ATRCqui aété au coeur de l'action, en assumant le "leadership" du développement régional; en outre, ellea permis à la population régionale d'y être associée.
L'ATR deCharlevoix a suprévoirà l'avance les grandes tendances du marché, en développant de nouvelles formes de tourisme, tel le tourisme de santé et le tourisme écologique.

En terminant, nous pouvons souligner quelques-uns des défis auxquels l'ATR de Charlevoix sera confrontée au cours des prochaines années dont, entre autres:

- stimuler les activités reliéesà la bonne marche de la Réserve de la Biosphère de Charlevoix;

- créerun parc national dans les HautesGorges de la rivière Malbaie;

- poursuivre le développement du parc des Grands-Jardins;

- étendre les frontières du parc marin jusqu'aux limites de Cap-à-l'Aigle, en confirmant à Saint-Siméon le rồle de siège administratif;

- assurer l'essor du produit hivernal par le développement de la station de ski du Massif ainsi que par le renforcement de produits secondaires comme la motoneige et le traîneau à chiens;

- faciliter la construction d'une salle de spectacles au Domaine Forget;

- permettre l'établissement d'un Pavillon de la Biosphère;

- prévoir la mise en place de bandes cyclables à l'íle aux Coudres;

- restaurer le quaide Baie-Sainte-Catherine.

La nature puissante de Charlevoix a inspiré les artistes peintres de plusieurs générations. Elle inspire maintenant les charlevoisiensqui, puisant à cette ressourceexceptionnelle, trouvent l'énergie pour soutenir tous ces développements durables et rentables. L'environnement est synonyme de tourisme dans Charlevoix devenue Réserve Mondiale de la Biosphère. Fière d'une tradition de villégiature plus que centenaire, la région de Charlevoix doit réussir à conserver ses avantages naturels pour les prochaines générations: le plan de développement s'avère certes un élément de la solution. 